\title{
Sensibilidade dos nematódeos gastrintestinais de caprinos ao ivermectin na região da Grande Porto Alegre - RS
}

\author{
Sensitivity of gastrointestinal nematodes to ivermectin in goats in the metropolitan \\ region of Porto Alegre, State of Rio Grande do Sul, Brazil
Mary Jane Tweedie de Mattos ${ }^{1}$, Carlos Marcos Barcellos de Oliveira ${ }^{1}$, Aline Silva Gouvea² \& Cíntia Bisogno Andrade ${ }^{2}$

\begin{abstract}
RESUMO
As infecções por nematódeos representam um entrave na caprinocultura, podendo levar a morte de animais. Para o seu controle é utilizado ivermectin em caprinos jovens. Este experimento foi realizado com o objetivo de verificar a eficácia deste principio ativo sobre caprinos naturalmente parasitados por nematódeos. Para isto, foram utilizados 12 animais sendo 6 medicados com ivermectin ${ }^{\circledR}$, na dose de $200 \mu \mathrm{g} / \mathrm{kg}$ v.o, e 6 não medicados (controle) Para a avaliação, utilizou-se o teste de redução na contagem de ovos e redução de larvas infectantes além da carga parasitária total (adultos). Amostras fecais foram coletadas no dia da medicação (dia zero), aos 7 e 14 dias após. No $14^{\circ}$ dia pós-medicação todos os caprinos foram sacrificados, para a coleta de helmintos e cálculo da carga parasitária total. Os resultados revelaram que o ivermectin reduziu em 93,24\% o número de ovos de Trichostrongyloidea em caprinos. Na cultura de larvas observou-se Haemonchus (41\%), Ostertagia (33\%) e Oesophagostomum (26\%), no dia zero e somente de Haemonchus (100\%) no 14ª dia após a medicação. No grupo controle, no dia da medicação foram observadas larvas de Haemonchus (71\%), Ostertagia (26\%) e Trichostrongylus (3\%). Estes mesmos gêneros foram identificados no $14^{\circ} \mathrm{dia}$, com os porcentuais de $75 \%, 23 \%, 2 \%$, respectivamente. A eficácia do ivermectin ${ }^{\circledR}$ frente as infecções por adultos de Haemonchus, Ostertagia e Trichostrongylus foi de 92,21\%, 100\% e 60,24\%, respectivamente, sendo que o gênero Haemonchus, representou $96 \%$ da população encontrada no grupo controle.
\end{abstract}

Descritores: caprinos, Haemonchus, Ostertagia,Trichostrongylus, Oesophagostomum, ivermectin.

\begin{abstract}
Infections by nematodes are some of the major drawbacks in the goat production industry and the use of Ivermectin in young animals, is one of the alternative strategies for the control of such parasites. The aim of this experiment was to evaluate the effect of Ivermectin ${ }^{\circledR}$ on the control of gastrointestinal nematodes in a group of six naturally infected goats. Another group with six animals received water (placebo) and was used as control. The fecal egg count reduction (FECR) test and the larval development assay (LDA) were used. Fecal samples were collected from the goats on the day of medication (day zero), at seven, and at fourteen days thereafter. All the goats were killed fourteen days after medication for collection of helminths and the total parasite load was calculated. The results showed that ivermectin given orally at $200 \mu \mathrm{g} /$ $\mathrm{kg}$ reduced the number of trichostrongylid eggs in goats by $93.24 \%$. The embryo development test revealed larvae of Haemonchus, Ostertagia and Oesophagostomum in respectively $41.33 \%$ and $26 \%$ of the animals on day zero and only larvae of Haemonchus in $100 \%$ of the goats fourteen days after medication. The control group revealed Haemonchus (71\%), Ostertagia (26\%) and Trichostrongylus (3\%) on the day of medication. These genera were also found fourteen days thereafter at the respective rates of $75 \%, 23 \%$, and $2 \%$. The efficacy of Ivermectin ${ }^{\circledR}$ against infections caused by the adult forms of Haemonchus, Ostertagia and Trichostrongylus were respectively 92.21\%, 100\% and 60.24\%. The genus Haemonchus, however, accounted for $96 \%$ of the population of helminths observed in the control group.
\end{abstract}

Key words: goats, anthelminthic, Haemonchus, Ostertagia e Trichostrongylus, Oesophagostomum, ivermectin.

${ }^{1}$ Setor de Helmintologia, Departamento de Patologia Clínica Veterinária, Faculdade de Veterinária (FAVET), Universidade Federal do Rio Grande do Sul (UFRGS). Av. Bento Gonçalves, 9090; 91540-000 Porto Alegre-RS. ${ }^{2}$ Acadêmicas do Curso de Medicina Veterinária/UFRGS. CORRESPONDÊNCIA: M. J. T. Mattos [mary.gomes@ufrgs.br; Fax +55 513316 7305]. 


\section{INTRODUÇÃO}

A produção de caprinos para a comercialização de leite ou de carne, é uma alternativa para pequenas propriedades, desde que se estabeleça um adequado manejo sanitário. Um dos entraves é a infecção com nematódeos gastrintestinais, principalmente do gênero Haemonchus $[6,8,18,26]$. A verminose caprina foi estudada no RS e observou-se parasitismo gastrintestinal, independentemente do sistema de criação [15]. Nas necropsias de caprinos provenientes da região da Grande Porto Alegre-RS, Haemonchus contortus e Trichostrongylus colubriformis foram os mais encontrados [4]. Em outro estudo, verificou-se que a verminose caprina ocorre durante todo o ano, sendo mais prevalentes Haemonchus e Ostertagia [18].

Para o controle de Haemonchus são indicados vários anti-helmínticos, entre eles o Ivermectin, registrado em mais de 60 países para uso em bovinos, ovinos, eqüinos, caprinos, suínos, bisões e cães [5, 9], e cuja eficácia em caprinos foi verificada na África do Sul [3,11,22], e no Brasil (Rio de Janeiro [19], Rio Grande do Sul [16], Bahia [1] e Alagoas [2]).

Os anti-helmínticos mais utilizados no controle de verminose caprina no RS, são levamisole (33\%), ivermectin (23\%), albendazole (19\%) e closantel (10\%), sendo o ivermectin mais utilizado para filhotes [17]. Pesquisas realizadas no RS demonstraram que a eficácia do Ivermectin é bastante variável, dependendo de vários fatores [16].

O objetivo deste experimento foi verificar a eficácia do Ivermectin sobre ovos, larvas e adultos de helmintos gastrintestinais de caprinos.

\section{MATERIAIS E MÉTODOS}

Inicialmente foram realizadas visitas a algumas propriedades rurais, com o objetivo de coletar fezes e informações sobre a frequiência de utilização de antihelmínticos. Os critérios adotados para a seleção dos animais incluídos neste experimento foram: ser machos, com similaridade de idade, que evidenciassem um número mínimo de ovos por grama de fezes (500) e a não utilização de qualquer anti-helmíntico, por um período mínimo de 30 dias.

Os caprinos selecionados foram transportados para a cabanha da Faculdade de Veterinária/UFRGS, em Porto Alegre-RS, onde permaneceram em adaptação alimentar por 15 dias, antes do início do expe- rimento. Nos primeiros três dias só receberam forragem de feno, duas vezes ao dia e, a partir daí receberam além de forragem de feno duas vezes ao dia, um concentrado não medicamentoso, uma vez ao dia, e água a vontade. A cabanha possui piso ripado e elevado do chão e as baias eram limpas diariamente. Os caprinos foram pesados e identificados com brinco numerado no dia da chegada.

Amostras fecais individuais foram coletadas diretamente do reto, identificadas e mantidas sob refrigeração até o processamento para a contagem de ovos e cultura para a identificação e contagem de larvas com o objetivo de constituir os lotes experimentais. No dia da medicação, os caprinos foram novamente pesados para o cálculo exato da dose do antihelmíntico.

Foram utilizados 12 caprinos naturalmente parasitados por nematódeos gastrintestinais, sem raça definida, com 4-5 meses de idade, com peso médio de $11,5 \mathrm{~kg}$, sendo constituídos dois grupos com seis animais cada um [25]. Um deles recebeu $200 \mu \mathrm{g} / \mathrm{kg}$ de ivermectin via oral e o outro (controle) recebeu apenas placebo (água) no mesmo volume e via de administração.

Amostras fecais de todos os caprinos foram coletadas no dia da medicação, aos sete e aos catorze dias após. As fezes foram coletadas diretamente do reto, identificadas e mantidas em refrigeração no Laboratório do Setor de Helmintologia da Faculdade de Veterinária/UFRGS. Posteriormente foram procedidas as contagens de ovos e as identificações de larvas infectantes respectivamente através dos Métodos de Gordon \& Whitlock (1939) e Roberts O'Sullivan (1950) [24]. No décimo quarto dia todos os caprinos foram sacrificados, para a coleta de helmintos gastrintestinais, após serem submetidos a um jejum sólido por 12 horas. Para a contagem de helmintos, foram consideradas amostras de $10 \%$ do abomaso e do intestino delgado e $100 \%$ do intestino grosso [24].

Para calcular a eficácia do ivermectin em relação a redução do número de ovos por grama de fezes (opg) foi utilizado o seguinte cálculo [7]:

$$
\% \text { de eficácia }=\frac{100(1-\text { média de opg no grupo medicado })}{\text { média de opg no grupo controle }}
$$

A eficácia do ivermectin sobre as populações de helmintos adultos presentes nos dois grupos foi avaliada através da fórmula [24]: 
A fecundidade média per capita das fêmeas

$$
\% \text { de eficácia }=\frac{\begin{array}{c}
\left(\mathrm{n}^{\circ} \mathrm{m} \text { édio de helmintos no grupo de controle }-\right. \\
\left.\mathrm{n}^{0} \mathrm{~m} \text { édio de helmintos do medicado }\right)
\end{array}}{\mathrm{n}^{0} \text { médio de helmintos no grupo controle }}
$$

foi calculada a partir da divisão do opg constatado no dia da necropsia pelo número de fêmeas adultas encontradas [12]. Os resultados foram avaliados através da análise da variança. Foram calculados os resíduos da análise da variança com o software SAS, e com esses resíduos foram calculados os coeficientes de correlação parcial utilizando o software SPSS [20].

\section{RESULTADOS}

A Tabela 1 mostra que houve uma redução de 93,24\% no número de ovos de Trichostrongyloidea entre o dia zero e o décimo quarto dia após a medicação, no grupo medicado. Em relação ao intervalo entre o dia zero e o sétimo dia foi observada uma redução no opg de 95,42\%. A identificação genérica das larvas infectantes no grupo medicado acusou a presença de Haemonchus com porcentuais de 41\%, $100 \%$ e $100 \%$ de Ostertagia com os porcentuais de $33 \%$, $0 \%, 0 \%$ e Oesophagostomum com os porcentuais de $26 \%, 0 \%$ e $0 \%$ nos dias zero, sete e catorze, respecti- vamente. Em relação ao grupo controle, no dia zero foram observadas larvas infectantes de Haemonchus (71\%) e Ostertagia (26\%) e Trichostrongylus (3\%). Nos dias sete e catorze, foram identificadas larvas infectantes de Haemonchus (81\%,75\%), Ostertagia $(17 \%, 23 \%)$ e Trichostrongylus (2\%,2\%).

Em relação à carga parasitária, houve maior número de Haemonchus no grupo controle do que no grupo medicado, como pode ser observado na Tabela 2. No grupo medicado, a carga parasitária total foi de 210 helmintos e, destes, 90,48\% eram Haemonchus. No grupo controle a carga parasitária foi de 2540 helmintos, sendo que $96,06 \%$ eram Haemonchus. A eficácia sobre as formas adultas de Haemonchus foi de 92,21\%. O número de fêmeas representou $57,89 \%$ do total de Haemonchus identificados no grupo medicado, e 55,32\% no grupo controle. A eficácia do ivermectin frente às infecções por adultos de Haemonchus, Ostertagia, Trichostrongylus foi, respectivamente, de 92,21\%, 100\% e de $60,24 \%$. Houve uma associação positiva direta entre a carga parasitária e opg $(\mathrm{r}=+0,487, \mathrm{p}<0,016)$.

Considerando os valores do último opg realizado (Tabela 1) e o número de fêmeas de helmintos encontrados nas necropsias (Tabela 2) foram determinados os índices de fecundidade de 2,08 para as

Tabela 1. Número de ovos de Trichostrongyloidea por grama de fezes, em caprinos após medicação com ivermectin (média e amplitude).

\begin{tabular}{cccc}
\hline Grupo & Zero Dia & Dia 7 & Dia 14 \\
\hline & 3658,33 & 125 & 250 \\
Medicado & $(500-14300)$ & $(0-300)$ & $(0-600)$ \\
Controle & 2525 & 2733,33 & 3700 \\
& $(500-8300)$ & $(500-8300)$ & $(200-19800)$ \\
\hline
\end{tabular}

Tabela 2. Carga parasitária de helmintos (total e média/animal) no abomaso de caprinos, 14 dias após medicação

\begin{tabular}{|c|c|c|c|c|c|c|c|c|c|}
\hline \multirow{2}{*}{ Grupo } & \multicolumn{3}{|c|}{ Haemonchus } & \multicolumn{3}{|c|}{ Ostertagia } & \multicolumn{3}{|c|}{ Trichostrongylus } \\
\hline & Machos & Fêmeas & Total & Machos & Fêmeas & Total & Machos & Fêmeas & Total \\
\hline \multicolumn{10}{|l|}{ Medicado } \\
\hline & 80 & 110 & 190 & 0 & 0 & 0 & 10 & 10 & 20 \\
\hline \multirow[t]{2}{*}{ Média } & 13,33 & 18,33 & 31,66 & 0 & 0 & 0 & 1,66 & 1,66 & 3,32 \\
\hline & $(0-60)$ & $(0-50)$ & & 0 & 0 & 0 & $(0-10)$ & $(0-10)$ & \\
\hline \multicolumn{10}{|l|}{ Controle } \\
\hline & 1090 & 1350 & 2440 & 20 & 30 & 50 & 30 & 20 & 50 \\
\hline \multirow[t]{2}{*}{ Média } & 1,86 & 225,00 & 406,66 & 3,33 & 5,00 & 8,33 & 5,00 & 3,33 & 8,33 \\
\hline & $(0-710)$ & $(0-980)$ & & $(0-10)$ & $(0-20)$ & & $(0-30)$ & $(0-20)$ & \\
\hline
\end{tabular}
com ivermectin. 
fêmeas do grupo medicado e de 2,64\% para as fêmeas do grupo controle. A diferença entre os grupos não foi significativa em nível de significância de 5\% através do Teste Estatístico não paramétrico Wilcoxon Rank Test. Não foram encontrados helmintos no intestino delgado e grosso

\section{DISCUSSÃO}

A eficácia do ivermectin ao ser calculada a partir da contagem de ovos de helmintos, no intervalo de 7 dias, após a medicação, foi de 95,43\%, o que é considerado como eficaz pela maioria dos pesquisadores [7,23]. Porém quando se avalia o intervalo de catorze dias, observa-se que este valor é inferior, 93,24\%, porcentual considerado por alguns autores $[7,23]$ como indicativo de início de resistência antihelmíntica e embora outros [25] considerem que até 90\% de redução é eficaz. Se o intervalo entre a aplicação e a avaliação do medicamento for inferior a 10 dias, poderá haver um resultado falso positivo, pois neste período pode ocorrer uma supressão da postura de ovos pela fêmea do helminto, que voltaria a realizar postura [23]. Isto já foi observado em caprinos parasitados por Ostertagia e medicados com ivermectin [13].

Neste experimento, pode ter ocorrido uma diminuição da postura temporária, o que justificaria o aumento posterior do opg. Nesta população de helmintos provavelmente já existam indivíduos resistentes ao ivermectin, principalmente do gênero Haemonchus. O ivermectin atua paralisando o helminto pelo aumento da permeabilidade muscular para os íons cloro [21]. Há pelo menos dois caminhos para ação do Ivermectin [10]: o principal é a ação sobre o canal íon glutamato, que tem sido associado ao bombeamento faringeano do helminto. $\mathrm{O}$ ivermectin afeta a motilidade e o bombeamento faringeano dos nematódeos adultos. Inibindo o bombeamento, leva a uma diminuição das reservas energéticas, pois o helminto passa muito tempo sem se alimentar, enquanto que a inibição da motilidade terá um efeito imediato sobre a capacidade do parasito de captar os nutrientes.

Pesquisas realizadas no Rio de Janeiro com ivermectin em caprinos parasitados por Haemonchus (95\%) e Cooperia (5\%) evidenciaram uma eficácia de $100 \%$ sobre estes helmintos, através da cultura de larvas [19], superior à verificada no presente experimento, para Haemonchus. Em relação aos demais helmintos identificados no presente experimento, o Ivermectin demonstrou uma baixa eficácia sobre as formas adultas de Trichostrongylus (60,24\%), e alta sobre Ostertagia (100\%). Em relação a este último gênero, os resultados se aproximam dos observados na África do Sul [3], em que a eficácia do ivermectin foi de $100 \%$, mas divergem dos outros resultados observados no RS [16]. Nestes últimos, foi verificada uma eficácia de $73,51 \%$, porém só sobre ovos e larvas infectantes. O gênero Trichostrongylus foi observado em baixa porcentagem no grupo medicado, nas necropsias, e não foi detectado nas coproculturas, provavelmente por não estar eliminando ovos ou eles não serem férteis.

No presente experimento, no grupo controle, $\mathrm{o}$ número médio de adultos de Haemonchus $(406,66)$ foi maior do que o encontrado (365) no município de Petrolina-PE [6] e, menor do que o relatado $(893,09)$ em outro experimento na Grande Porto Alegre-RS. [4].

\section{CONCLUSÃO}

Os resultados encontrados nesta pesquisa mostram que o gênero Haemonchus está presente em maior porcentagem do que Ostertagia e Trichostrongylus. O emprego de ivermectin em caprinos requer um monitoramento periódico, através da contagem de ovos e cultura de larvas infectantes, pois através deste experimento observou-se que alguns nematódeos resistiram à aplicação de ivermectin. 


\section{REFERÊNCIAS}

1 Barreto M.A, Almeida M.A., Silva A, Rebouças I.\& Mendonça L.R. 2002. Eficácia anti-helmíntica de cloridrato de levamisole, Albendazole e Ivermectin em caprinos, na região semi-árida da Bahia. In: Anais do XII Congresso Brasileiro de Parasitologia Veterinária (Rio de Janeiro, Brasil). 1 CD.

2 Bispo M. S., Faustino M.A.G, Lima M.M., Oliveira G.A, Ramos C.A \& Bispo C.A.S.2002. Avaliação do tratamento antihelmíntico com oxfendazole e ivermectina em rebanho caprino do Instituto Xingó-município de Piranhas-Alagoas In: Anais do XII Congresso Brasileiro de Parasitologia Veterinária (Rio de Janeiro, Brasil). 1 CD.

3 Borgsteede F.H.M., Pekelder J.J.\& Dercksen D.P. 1996. Anthelmintic resistant nematodes in goats in the Netherlands.Veterinary Parasitology. 5: 83-87.

4 Cardoso J.L.S. \& Oliveira C.M.B. 1993. Fauna parasitária de caprinos da grande Porto Alegre. Revista Brasileira de Parasitologia Veterinária. 2: 57-60.

5 Cerkvenik V., Grabnar I., Skubic V., Doganoc D.Z, Beek W.M.J, Keukens H.J., Kogorok M.D.\& Pogagnik M. 2002. Ivermectin pharmacokinetics in lactating sheep. Veterinary Parasitology.104: 175-185

6 Charles T.P. 1989. Seasonal prevalence of gastrointestinal nematodes of goats in Pernambuco State, Brasil. Veterinary Parasitology. 30: 335-343.

7 Coles G. C., Bauer C., Borgsteede F.H.M, Geerts S., Klei T.R. \& Taylor M.A. 1992. World Association for the advancement of Veterinary Parasitology (WAAVP) methods for the detection of anthelmintic resistance in nematodes of veterinary importance. Veterinary Parasitology. 44: 35-44.

8 Costa C.A.F., Vieira L.S. \& Berne M.E.A. 1991. Influência das instalações de pernoite, do tipo de pastagem e da suplementação volumosa sobre o parasitismo por nematódeos em caprinos. Pesquisa Agropecuária Brasileira. 26: 521-533.

9 Di Netta J. 1989. List of registrations.Appendix III to Campbell W.C. (Ed). Ivermectin and abamectin. New York: Springer-Verlag, pp. 344-366.

10 Gill J.H \& Lacey E. 1998. Avermectin/milbemycin resistance in trichostrongyloid nematodes.International Journal for Parasitology. 28: 863-887.

11 Hoyt P.G., French J.E., Miller J.E., Williams J.C., Hackett G.E., Kearney M.T. \& Hoyt, M.J. 1992. Evaluation of ivermectin against experimental infections of Haemonchus contortus and Trichostrongylus colubriformis in goats. Veterinary Parasitology. 42: 257-257.

12 Houdijk J.G.M., Kyriazakis I., Jackson F., Huntley J.F.\& Coop R.L. 2000. Can an increased intake of metabolizable protein affect the periparturient relaxation in immunity against Teladorsagia circumcincta in sheep. Veterinary Parasitology. 91: 43-62.

13 Jackson F. 1993. Anthelmintic resistance-the state of play. British Veterinary Journal. 149: 123-138.

14 Lacey E. 1998. Avermectin/milbemycin resistance in trichostrongyloid nematodes. International Journal for Parasitology. 28: 863-877.

15 Mattos M.J.T., Schmidt V., Bastos C.D \& Arosi A. 1992. Flutuação de ovos de nematódeos gastrintestinais de caprinos em diferentes sistemas de criação.In: XI Congresso Estadual de Medicina Veterinária (Gramado, Brasil). p.52.

16 Mattos M.J.T., Germer M.\& Castro E.S.D. 1997. Eficácia do ivermectin sobre endoparasitos de caprinos, no RS. In: XI Congresso Brasileiro de Medicina Veterinária (Gramado, Brasil). p.198.

17 Mattos M. J. T. \& Castro E. S.D. 2002. Utilização de anti-helmínticos no controle de verminose caprina no Estado do Rio Grande do Sul. A Hora Veterinária. 130: 52-54.

18 Mattos M.J.T. \& Bastos C. D. 2000. Parasitoses de caprinos no município de Viamão-RS. Revista Brasileira de Ciencia Veterinária. 7 (supl): 217.

19 Mesquita M.\& Hees S.J. 1992. Teste comparativo da eficácia de ivermectina oral em caprinos perante um outro antihelmintico de uso rotineiro. A Hora Veterinária. 66: 23-26.

20 Montgomery D.C. 1997. Design and Analysis of Experiments. New York: John Wiley \& Sons, 704 p.

21 Ranjan S., Wang G.T., Hirschlein C.\& Simkins K.L. 2002. Selection for resistance to macrocyclic lactones by Haemonchus contortus. Veterinary Parasitology.103: 109-117.

22 Swan G.E. \& Gross S.J. 1985. Efficacy of ivermectin against induced gastrointestinal nematode infections in goats.Veterinary Record. 17: 147-149.

23 Taylor M.A, Hunt K.R. \& Goodyear K.L. 2002. Anthelmintic resistance detection methods. Veterinary Parasitology. 103: $183-194$. 
24 Ueno H. \& Gonçalves P.C. 1998. Manual para Diagnóstico das Helmintoses de Ruminantes. 4.ed. Tóquio:Japan International Cooperation Agency, 166p.

25 Vercruysse J., Holdsworth P., Letonja T., Barth D., Conder G., Hamamoto K. \& Okano K. 2001. International harmonisation of anthelmintic efficacy guidelines. Veterinary Parasitology. $96: 171-193$.

26 Vieira L. \& Calvacante A. C. R. 1998. Resistência anti-helmintica em nematódeos gastrintestinais de caprinos. Revista Brasileira de Medicina Veterinária. 20: 112-117. 